\title{
A sample chamber for in-situ high-energy X-ray studies of crystal growth at deeply buried interfaces in harsh environments
}

\author{
A.E.F. de Jong ${ }^{\mathrm{a}, \mathrm{b}}$, V. Vonk ${ }^{\mathrm{b}, \mathrm{c}}$, V. Honkimäki ${ }^{\mathrm{a}}$, B. Gorges ${ }^{\mathrm{a}}$, H. Vitoux ${ }^{\mathrm{a}}$, E. Vlieg ${ }^{\mathrm{b}}$ \\ ${ }^{a}$ The European Synchrotron Radiation Facility, BP 220, F-38043, Grenoble Cedex 9, France \\ ${ }^{b}$ Institute for Molecules and Materials, Radboud University Nijmegen, Heyendaalseweg 135, 6525AJ Nijmegen, The Netherlands \\ ${ }^{c}$ DESY Nanolaboratory, Deutsches Elektronen-Synchrotron (DESY), Notkestraße 85, D-22607 Hamburg, Germany
}

\begin{abstract}
We introduce a high pressure high temperature chamber for in situ synchrotron x-ray studies. The chamber design allows for in situ studies of thin film growth from solution at deeply buried interfaces in harsh environments. The temperature can be controlled between room temperature and $1073 \mathrm{~K}$ while the pressure can be set as high as 50 bar using a variety of gases including $\mathrm{N}_{2}$ and $\mathrm{NH}_{3}$. The formation of $\mathrm{GaN}$ on the surface of a $\mathrm{Ga}_{13} \mathrm{Na}_{7}$ melt at $1073 \mathrm{~K}$ and 50 bar of $\mathrm{N}_{2}$ is presented as a performance test.
\end{abstract}

Keywords: A1. X-ray diffraction, A1. Interfaces, A3. Liquid phase epitaxy, B1. Gallium compounds, B1. Nitrides, B2. Semiconducting III-V materials

\section{Introduction}

Interfaces are encountered on a daily basis. Examples are the formation of an interface between immiscible fluids, like oil and water; artificially bonded wafers, like microelectromechanical systems (MEMS); a solid in contact with its melt, like water on ice; a metal in contact with a semiconductor, like a Schottky contact; growth of single crystals from a liquid source, like gallium nitride $(\mathrm{GaN})$ liquid phase epitaxy (LPE) growth. The 'buried' interfaces between two bulk systems, as described above, are often causing interesting phenomena. Examples are enhanced liquid density [1], liquid order [2] or liquid layering [1, 3, 4]. These phenomena are important factors with respect to the mass transport towards the growing interface and therefore growth speed and crystalline quality. Surface x-ray diffraction is capable of resolving a complete 3D structure of the interfaces described above. However, the structure at the interface is difficult to investigate with conventional $\mathrm{x}$-ray energies $(E \approx 10 \mathrm{keV})$ due to the attenuation by the material to be penetrated.

The last two decades however, the creation of proper instrumentation $[5,6]$ at high brilliance high-energy $\mathrm{x}$-ray sources allowed scientists to start exploring these more realistic systems and more complex interfacial phenomena [7-10].

Many interfaces in crystal growth are formed in harsh conditions often combining high pressure, high temperature and dangerous gasses or liquids to form the required material. A suitable sample environment is therefore key to investigate the buried interfaces formed during crystal growth in such environments.

This paper presents a reusable batch reactor specifically designed for in-situ studies of growing, heated and pressurized buried interfaces in corrosive environments by means of high-energy x-ray reflectivity (XRR) and crystal truncation rod (CTR) measurements. The use will initially be limited to GaN growth using the Na-flux technique. However, this is only one of the experiments that can be envisioned.

To the best of our knowledge this chamber is unique in that it is the first large volume cell designed with a carbon fiber window that is capable of surface diffraction experiments at elevated pressure and temperature using corrosive gasses and liquids. Previous pressure cells for XRR were not made for temperatures over $360 \mathrm{~K}$ nor corrosive materials ([9] and references therein). Pressure cells for CTR measurements are even more scarce [11], and to the best of our knowledge not available for the combination we present here. 


\section{Interface diffraction from deeply buried inter- faces}

Performing diffraction from deeply buried interfaces means that one of the materials forming the interface has to be penetrated. This implies the use of high energy $\mathrm{x}$-rays, because the $\mathrm{x}$-ray attenuation coefficient $(\mu)$ rapidly decreases with increasing x-ray energy: $\mu(E) \approx$ $E^{-3}$.

An additional benefit of $\mathrm{x}$-rays is that for increasing $\mathrm{x}$ ray energy the scattering angle of a specific signal will decrease. This means that for high energy surface $\mathrm{x}$ ray diffraction (SXRD) signals will be found relatively close to the (000) reflection which reduces the demands for $\mathrm{x}$-ray windows in chambers.

A disadvantage of (high energy) $\mathrm{x}$-rays is that they are not intrinsically surface sensitive and the bulk signal can drown the surface signal. By selecting surface sensitive reflections and additionally setting slits to suppress parasitic scattering and increase the signal to noise ratio this problem is largely overcome. Additionally one can increase the signal to noise ratio further by choosing small incident angles for CTR measurements, typically equal to or lower than the critical angle of reflectivity ensuring total reflection of the incident beam. The small resulting incident angle can lead to complications. Setting an incident angle lower than or equal to the critical angle has a consequence for the required surface quality. The use of high energy $x$-rays means that the critical angle of reflectivity is very small, typically around $0.03^{\circ}$ at $70 \mathrm{keV}$. Even with a focussed $\mathrm{x}$-ray beam, a small incident angle means illumination of a big surface (e.g. a vertically focussed beam of $8 \mu \mathrm{m}$ at $0.03^{\circ}$ incidence angle will illuminate approximately $15 \mathrm{~mm}$ of the sample surface). For this reason the sample surface has to be polished to an extremely smooth (surface roughness) and flat (wavyness, curvature) finish. A detailed description on SXRD is given elsewhere [12, 13].

The chamber we describe in this paper is designed primarily for transmission SXRD at high energies. However, the available exit angle for scattered X-rays is chosen so that bulk powder diffraction and bulk liquid scattering, as presented in this paper, are optional alternative techniques. Additionally the chamber can be used with lower photon energies. Compared to $70 \mathrm{keV}$ photons where the transmission through the chamber equals $85 \%$, the transmission at $20 \mathrm{keV}$ is still $68 \%$. Note however that the incident and exit angles available might become an issue when utilizing x-rays of lower energy.

\section{Experimental requirements and chamber design}

\subsection{The Beamline}

The chamber is developed for use on the high energy micro diffraction (HEMD) stage $[5,6]$ at the high energy beamline (ID15) of the European Synchrotron Radiation Facility (ESRF). The HEMD setup is presented in figure 1.
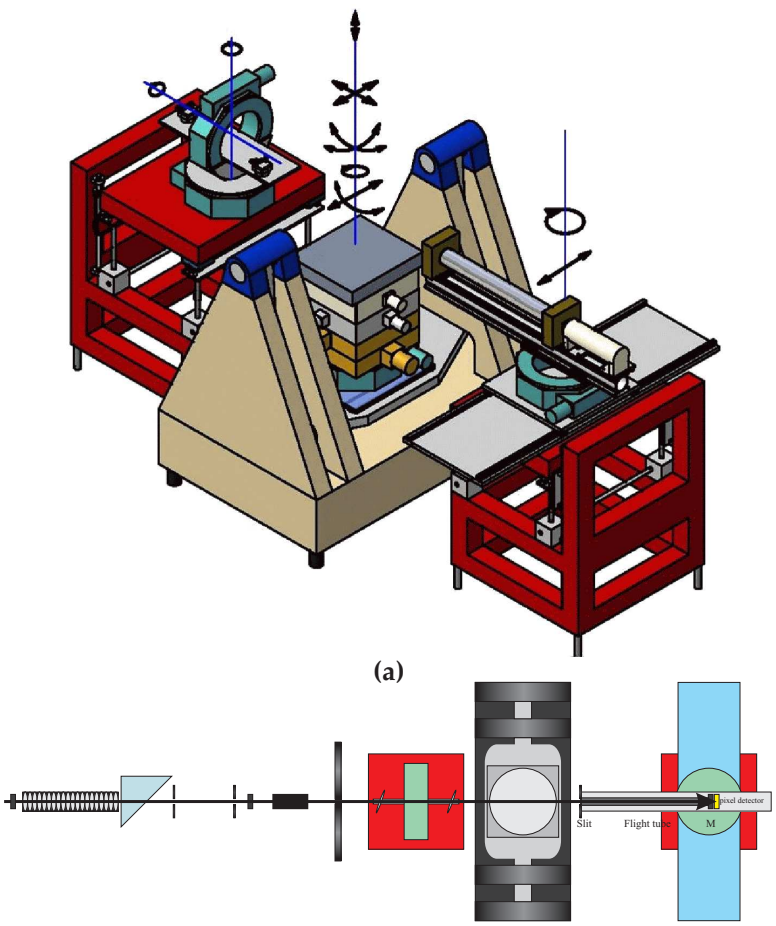

M CRL Abs Slits M FS LW L-OPT HEMD Detector

(b)

Figure 1: (a): Schematic of the high energy micro diffraction (HEMD) setup at beamline ID15 of the ESRF showing from left to right the optics for liquid interface/surface studies (L-OPT in (b)), the HEMD and the detector table (Detector in (b)).

(b): Top view of beamline setup with $\mathrm{M}=$ monitor diode, $\mathrm{CRL}=$ compound refractive lenses, Abs = PMMA Absorber, FS = fast shutter, LW = lead wall, L-OPT = optics for liquid interface/surface studies, HEMD = high energy micro diffractometer.

To improve data acquisition the HEMD setup has been equipped with a 2D Maxipix detector with Cadmium Telluride (CdTe) direct detection sensor [14]. Such a detector allows for data collection in the so-called stationary mode [15]. Both the scattering signal and the background can be determined from a single image and there is no need for rocking the crystal. This speeds up data collection tremendously. Combining the use of a pixel detector, the possibility of XRR and CTR measurements, and the use of high energies $(E \geq 70 \mathrm{keV})$ 
gives fast access to complete 3D structures of deeply buried interfaces.

\subsection{Chamber Requirements}

The semiconductor Gallium Nitride $(\mathrm{GaN})$ can be grown using several methods. One of the main techniques to grow high quality GaN crystals used at the moment is growth from supercritical ammonia with low quality crystalline GaN as nutrient, so-called ammonothermal growth. This technique requires pressures and temperatures above 100 bar and $650 \mathrm{~K}$ respectively. Another technique is the growth using nitrogen gas instead of ammonia which demands pressures up to 2000 bar and temperatures as high as $2000 \mathrm{~K}$. In both cases the pressure forms a problem since a feasible setup with a $360^{\circ}$ accessible $x$-ray window for in-situ x-ray diffraction is not easily achievable without risking unsafe working environments. However, there is a third growth technique in which the pressure requirements are drastically reduced. GaN growth can be performed at significantly lower pressures $(P \leq 50$ bar in the case of $\mathrm{N}_{2}$ use, or $P \leq 5$ bar in the case of $\mathrm{NH}_{3}$ use) and temperatures up to $1073 \mathrm{~K}$ when adding sodium to the liquid gallium $[16,17]$. Using this last technique, the so-called Na-flux LPE method, a growth chamber for in-situ $x$-ray diffraction is feasible.

Up to now most experiments to form $\mathrm{GaN}$ using the Naflux method are performed in stainless steel tubes which are sealed by welding. After the growth is finished the tubes are cut open to remove the newly formed material and the highly corroded tubes are thrown away.

We set forth to create a reusable chamber (figure 2) for in situ optimization of reaction parameters for the $\mathrm{GaN}$ Na-flux growth technique.

The chamber is developed keeping in mind that it has to be transported in and out of a glovebox to ensure a safe transfer of sodium and liquid gallium into and out of the chamber. For this reason the chamber dimensions were restricted to that of the antechamber of the glovebox intended for use which resulted in maximum dimensions of the chamber equal to $268 \mathrm{~mm}$ height (substrate heater fully up) and $200 \mathrm{~mm}$ radius.

What follows is a list of the different requirements and the solutions chosen for the chamber.

Pressure and $x$-ray window. Given that Na-flux LPE $\mathrm{GaN}$ growth can be performed effectively at pressures up to 50 bar, the chamber is designed in such a way that the limiting factor is given by the maximum pressure, $P_{\text {max }}$, that can be applied to the x-ray window of the cell.

Often X-ray windows are made of beryllium $(\mathrm{Be})$ or

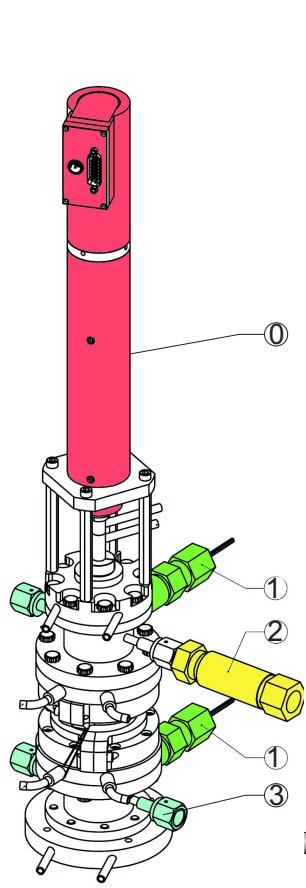

(a)

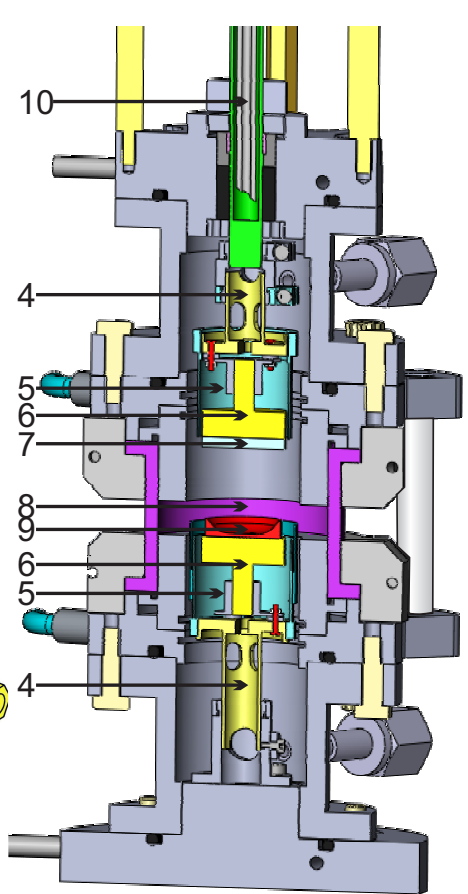

(b)
Figure 2: (a): Schematic showing the actuator (0) for moving the substrate, two high pressure feed troughs (1) (max pressure 250 bar), one safety valve (2) (max pressure 50 bar), 2 gas inlets and 1 gas outlet connector (3). (b): Section view of the furnace's interior showing: heater support (4), heater (5), heat shield (6), substrate (7), carbon fiber window (8), crucible (9), water cooled connection towards actuator (10). 
Kapton to ensure high transparency. Since liquid gallium is used in the chamber a Be window is unsuitable because gallium reduces beryllium to a powder. Also Kapton is not a good candidate because it will be decomposed by ammonia gas and cannot withstand high tensile forces without deforming.

For the above reasons carbon fiber was chosen as a material for the $\mathrm{x}$-ray window. Carbon fiber is transparent for x-rays of relatively high energy, it is mouldable in virtually any shape and can withstand high tensile stress without structural failure. It does give some small angle and wide angle scattering but this can be subtracted from the signals of interest. Since carbon fiber sheets are held together with an epoxy resin, the resin has to be inert in the conditions applied to the window. We used three methods to achieve this. Firstly, by coating the inside of the window with boron nitride paint the window does not suffer from contact with hot ammonia gas while it remains transparent for x-rays. Secondly, gallium does not deteriorate the quality of the window. And lastly, the carbon fiber epoxy is much more resilient against ammonia attack if the window is not getting too hot. Since the window is directly cooled by the water cooled flanges it is attached to, only the small exposed region is heated considerably. By using two high volume ventilators the exposed part of the $\mathrm{x}$-ray window can be kept well below $373 \mathrm{~K}$ even if the chamber is working at $1073 \mathrm{~K}$ and 50 bar for a long time.

The chamber has been successfully tested using a hydraulic press up to a pressure of 150 bar. This test was performed without attaching the actuator and without internal components and it assures the safe use at the routine pressure of 50 bar. The safe use is additionally assured by 4 metal bolts (one of them is visible on the right in figure $2 \mathrm{~b}$ ) which slightly compress the carbon fiber window to reduce the axial load on the glue holding the window $(6500 \mathrm{~kg}$ at $150 \mathrm{bar})$. A disadvantage of the bolts is the blocking of $\mathrm{x}$-ray windows over a range of 6 degrees per bolt. Depending on the symmetry of the crystal under investigation the choice for 3 or 4 bolts circumvents this problem partly.

The radial load on the exposed part of the window (10 $\mathrm{mm}$ heigh, $52 \mathrm{~mm}$ inner diameter) at 150 bar is approximately $2500 \mathrm{~kg}$. Combining these numbers with a hot and corrosive environment prompted for a large overcapacity in the accepted maximum tensile load on the carbon fiber window. Initial calculations showed that a window thickness of $0.5 \mathrm{~mm}$ should be enough to contain 150 bar of pressure at room temperature. By choosing a thickness of $2 \mathrm{~mm}$ a safe environment in a hot and corrosive environment should be achievable in terms of the window. The manufacturing of a $2 \mathrm{~mm}$ thick carbon fiber tube with rims on top and bottom posed a problem, however. The rim on top and bottom are present to reduce the axial stress on the glue and to provide a good place to clamp the window. Thin rims are however difficult to fabricate, especially when they should reduce axial force. By increasing the thickness of the window from 2 to $4 \mathrm{~mm}$, which consequently increased the amount of material available to form the rims, this problem could be solved. Additional information on the fabrication of the window (type of glue, type of epoxy, carbon weave pattern, et cetera) was kept secret by the manufacturer [18].

All following safety tests were performed with the full chamber assembled. First a temperature test using 1 bar argon at $1123 \mathrm{~K}$ for 2 hours showed that heaters, thermocouples, cable protection, pressure seals and X-ray window were unaffected. An additional pressure test was performed with a mix of $60 \%$ nitrogen and $40 \%$ ammonia at 5 bar and $1023 \mathrm{~K}$ without sodium and gallium to test the compatibility of the chamber with corrosive atmospheres.

Thereafter, the carbon fiber window was tested for radiation damage by irradiating $1 \mathrm{~mm}^{2}$ of the window for 1 week with $10^{12} 38 \mathrm{keV}$ photons/s. This treatment introduced a slight discoloration of the inner epoxy layer which disappeared on wiping with a tissue. Subsequent scans of the carbon fiber comparing irradiated and nonirradiated parts show that the window behaves as an amorphous material without a change in absorption and diffraction on the heavily irradiated part. Compared to the more common beryllium or sapphire $\mathrm{x}$-ray windows the carbon fiber window is reducing the price while strength and chemical stability are at least equal. Additionally, should the carbon fiber break, it will rip open, reducing damage to other parts while beryllium or sapphire will fail catastrophically.

Temperature. Basic working temperatures for effective Na-flux LPE GaN growth are reported up to $1073 \mathrm{~K}$. For this reason the chamber is designed to work at a maximum temperature of $1123 \mathrm{~K}$ at the maximum pressure. Increasing the temperature further will overheat the carbon fiber window. However, if the pressure is reduced to approximately 25 bar the temperature can be increased to the maximum working temperature of the heaters, $1273 \mathrm{~K}$ (heatwave labs model 101275-27). Furthermore both heaters can be controlled independently to allow the use of a temperature gradient. Tests show that the applied gradient can be as high as $600 \mathrm{~K}$ over 1 $\mathrm{cm}$. 
Corrosion protection. The chamber is made from inconel 600 steel (Ni $\geq 72 \%$, Cr 14-17\%, Fe 6-10\%) to prevent any corrosion by ammonia, evaporated sodium, liquid gallium or any reaction product (e.g. liquid sodium hydroxide or hydrogen gas). Park et al. used inconel as crucible material for GaN LPE growth [19] which resulted in traces of $\mathrm{Cr}$ and $\mathrm{Fe}$ in the grown $\mathrm{GaN}$. Since we do not have a direct contact between inconel and metal flux the amount of impurities in GaN originating from inconel should be significantly lower than reported by Park et al.

All cables and feed throughs are coated by Teflon to prevent attack by hot ammonia gas. The temperature of the cables does not exceed $373 \mathrm{~K}$ which ensures chemical stability of the Teflon. However, the amount of fluorocompounds present in the chamber is a cause of concern if one wants to perform other types of experiments.

\subsection{The Chamber}

The carbon fiber x-ray window is marked pink in figure $2 \mathrm{~b}$. It is glued to both the inner (dark grey) and outer (light gray) metal parts. Additionally the outer metal parts are designed to press inwards, squeezing the window against the inner metal parts to further prevent leaking. All other seals in the chamber use kalrez O-rings (black) that are close to a water cooling channel to prevent degradation of the rubber due to overheating.

Loading the cell with substrate and metals can be done by unbolting several screws (light yellow). A sample change can therefore be done without disassembling the window connections. The crucible (9) can be loaded with the required materials while on the top heater a substrate can be mounted (7) which has a diameter up to 1 inch. The substrate can be glued or clamped on an inconel plate that is attached to the physical heater. The inconel plate protects the heater from being corroded by gallium, sodium and sodium hydroxide and is easily exchanged.

There are two electrical feed throughs (Spectite WFS $1 / 4$ "NTP with exchanged wire coating, marked (1) in figure 2 a), one on the top and one on the bottom designed to withstand a pressure of 250 bar. The feed throughs are using a teflon plug to seal in the pressure. Additionally, there are four multi connectors of which one is reserved for a safety valve, marked (2), which opens at 50 bar. The others, marked (3), can be used for gas lines and a pressure gauge. On the top, marked (0), there is a heavy duty actuator (PI M-238.5PL) capable of moving the substrate reproducibly while the cell is under pressure. This specific actuator was chosen because of its maximum load capacity of $400 \mathrm{~N}$. The design of the chamber then allowed for a water cooled tube (marked (10) in figure $2 \mathrm{~b}$ ) with outer diameter of $10 \mathrm{~mm}$ which at 50 bar generates a pushing force just under 400 N. Four consecutive kalrez gaskets (Dupont KF-3074), compressed by a metal plate on top of the chamber, allow reproducible movements of the tube on a micrometer scale while gasses remain contained. The whole actuator and its support structure can be dismounted for easy manipulation and transportation while preparing for experiments. A picture of the whole cell in operation is shown in figure 3 .

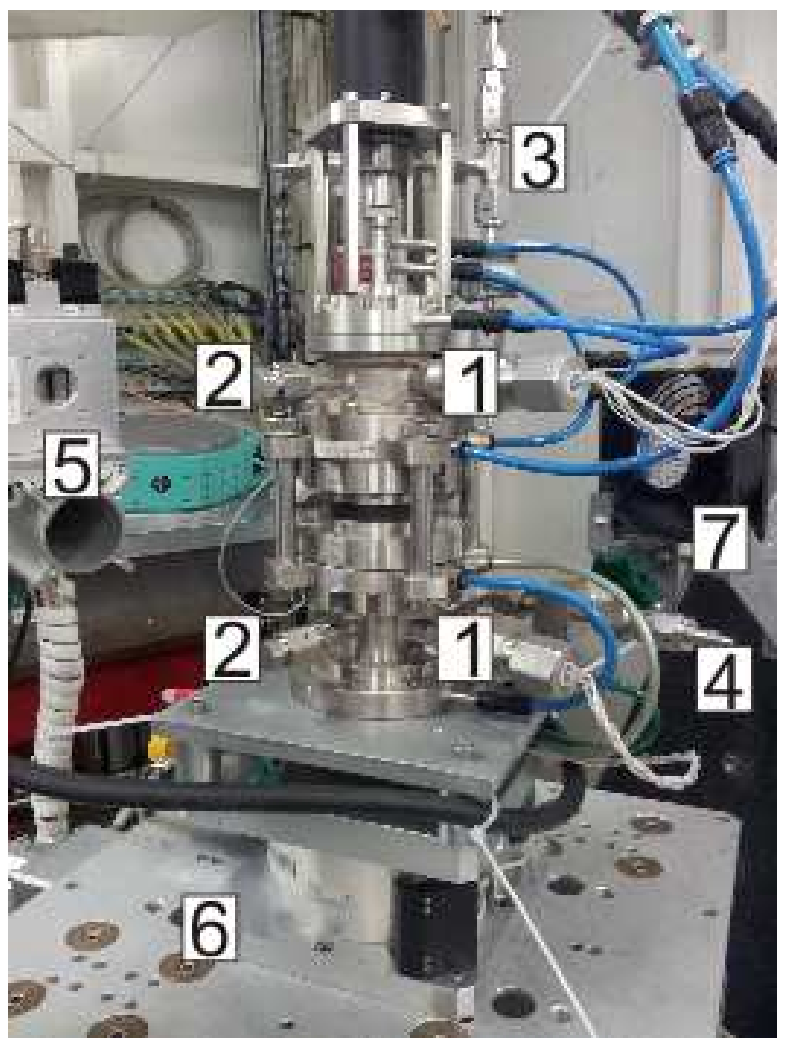

Figure 3: The cell undergoing safety tests for pressure, temperature and corrosion at the ESRF high energy beamline. Shown are 1: Electrical feed throughs, 2: gas connectors, 3: gas inlet, 4: gas outlet, 5: detector arm, 6: HEMD diffractometer tower, 7: high volume fan.

The whole furnace can be cleaned easily, if needed, after an experiment. The furnace, except for the crucible containing leftover sodium, can be immersed in demineralized water to remove any sodium hydroxide residue that could form on the furnace walls. Baking at $373 \mathrm{~K}$ in an autoclave will ensure a dry environment for a following experiment. 


\section{Experiment: Na-flux gallium nitride growth at $1073 \mathrm{~K}$}

Production of $\mathrm{GaN}$ is possible from the gas phase but yields are low due to large defect densities and crack formation. At the moment high quality $\mathrm{GaN}$ wafers grown in supercritical ammonia are commercially available with diameters up to $1 \mathrm{inch}$. The production is however very demanding. The demands for production of GaN using Na-flux growth are potentially much easier to attain while the quality of the grown material is much better than achievable with gas phase growth. For these previous reasons LPE GaN is a likely candidate to compete with expensive high pressure $\mathrm{GaN}$ growth techniques for parts of the market where extreme high quality bulk crystals are not needed (e.g. LEDs, lasers). The quality of the bulk GaN crystal is largely dictated by the growing interface. The development of this interface is largely unknown in the case of GaN. At high pressure it is difficult to make an accessible optical path for interface characterization while gas phase reactors are often too bulky to manipulate in their entire form. For these reasons in situ characterization has been limited. For gas phase growth, some information on the amount of material grown and very little on its overall quality can be extracted with the in situ measurement of reflected light from a laser parallel to the surface normal of the substrate [20, chap. 3.4.3]. With conditions of 50 bar, $1073 \mathrm{~K}$, liquid sodium and liquid gallium, the in-situ characterization of GaN Na-flux growth is possible with our chamber. Additionally our chamber allows easy investigation of growth parameters like temperature, pressure and gas composition which normally requires growth of multiple samples and extensive, often destructive, characterization afterwards.

The experiment. The experiment was performed at ESRF beamline ID15B using an x-ray energy of 87.3 $\mathrm{keV}$. Solid $\mathrm{Ga}_{13} \mathrm{Na}_{7}$ was used to fill the crucible. In order to perform a controlled and well defined experiment the temperature was first increased in 1 bar of Argon gas. When increasing the temperature to $1073 \mathrm{~K}$ it was observed that the metal mix became liquid at a temperature close to $835 \mathrm{~K}$ in agreement with literature [21]. After reaching $1073 \mathrm{~K}$ the pressure was increased from 1 bar Ar to 10 bar $\mathrm{N}_{2}$ which was left to equilibrate for 3 hours. Growth of $\mathrm{GaN}$ at this point is possible according to [17], but very unfavorable. Even on the interface between $\mathrm{N}_{2}$ gas and GaNa mix we did not observe any change in the scattered intensity during the equilibration time as is visible in top left figure 4.

After increasing the pressure to 50 bar, the first image

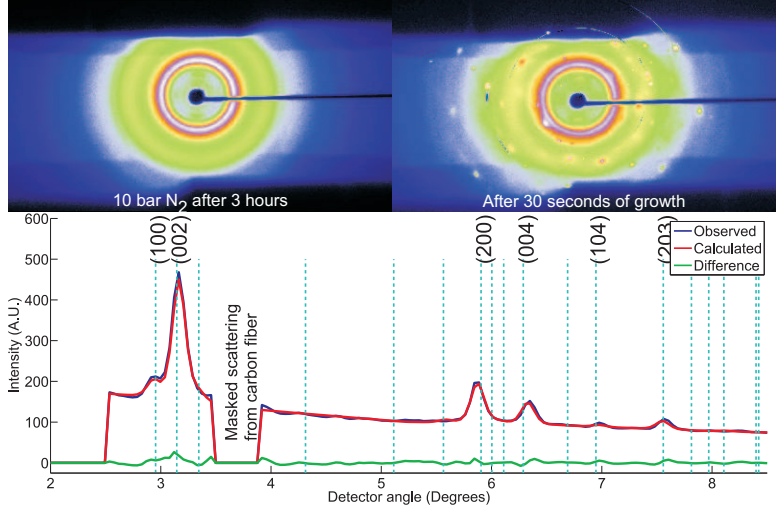

Figure 4: Top left: Diffraction pattern taken just below the interface between 10 bar $\mathrm{N}_{2}$ and $\mathrm{Ga}_{13} \mathrm{Na}_{7}$ mix. The intense circle is displaying the first liquid diffraction peak of the GaNa mix. Top right: Diffraction pattern on the interface between 50 bar $\mathrm{N}_{2}$ and $\mathrm{Ga}_{13} \mathrm{Na}_{7}$ mix showing Bragg spots coming from polycrystalline GaN. Bottom: A standard le Bail fit on the middle image shows intensity on several GaN Bragg positions. The sample was not rotated during the exposure explaining the absence of other Bragg peaks.

(top right in figure 4) immediately showed Bragg intensity indicating growth of crystalline material on the interface between gas and liquid. The Bragg spots became more intense but did not move in consecutive images which indicates growing polycrystalline material at the edge of the crucible, as expected from literature [22]. A le Bail fit (bottom figure 4) shows that the Bragg peaks observed are from hexagonal $\mathrm{GaN}$ which indicates that $\mathrm{GaN}$ can be grown with the furnace.

Additional use of a 1 inch diameter GaN seed crystal (e.g. an MOCVD GaN buffer layer on 1 inch sapphire, or an ammonothermally grown 1 inch wafer) which is placed on the top heater in the chamber can act as a smooth surface on which to perform surface diffraction. A well collimated $70 \mathrm{keV}$ X-ray micro-beam can then impinge the crystal from one side (see figure 5) and penetrate unrefracted down to the buried interface [5]. The reflected beam which carries information from the buried interface leaves the crystal on the other side after a full transmission and may be detected in a standard way. A small correction should be applied for the change in absorption path length through the whole crystal however. A simple measurement of the total attenuation factor of the direct beam by the crystal, multiplied by the path length at specific angles of incident and exit will suffice. Furthermore, when performing offspecular CTR measurements the crystal rotates which implies an additional change in absorption path length throughout one scan unless circular crystals are used. The chamber is primarily designed for interface diffrac- 


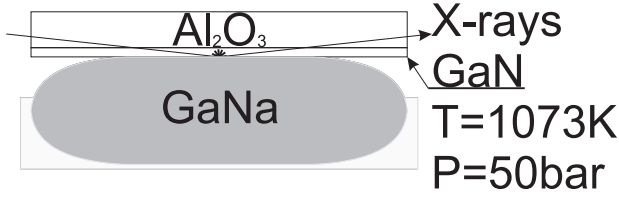

Figure 5: Schematic of the envisioned high energy surface x-ray diffraction experiment. X-rays will pass through the side of the crystal and exit on the other.

tion at the ID15A end station. However, the images collected at the ID15B end station, which are presented here, show the feasibility of the foreseen experiments in this chamber in one image. Numerous Bragg peaks are reacheable and not masked by the chamber. The parasitic scattering visible in the images is mainly originating from the carbon fiber window and is not causing problems for experiments at the ID15A end station unless collimation of the exiting beam is not discriminating between the origins of the scattering (front carbon fiber, sample, rear carbon fiber), be it because the slits are too far from the sample, too close to the detector or not closed enough. Recently the solid liquid interface between $\mathrm{GaN}$ and $\mathrm{Ga}$ at high pressure and high temperature has been successfully investigated using the HEMD device at the ID15A beamline in combination with the chamber presented in this paper. Those results will be presented elsewhere.

The deterioration of the chamber has been evaluated after several weeks of continued use. We have not found any damage to the chamber due to the harsh experimental conditions except for the two inconel plates holding the sample and crucible. These easily exchanged plates, intended to protect the heaters, should be replaced after approximately 6 months of continuous operation and can be cleaned in between experiments by slightly sanding them down to remove rough parts of the plate.

\section{Conclusion}

A new experimental setup, enabling high energy surface $x$-ray diffraction and high energy $x$-ray reflectivity from deeply buried, pressurize interfaces at elevated temperatures in harsh environments has been described. To demonstrate the potential of the new chamber, we have shown that GaN can be formed in the chamber using the Na-flux method. We have shown that growth occurs on the crucible holding the liquid metal mix. Further experiments and analyses are expected to provide new molecular scale information on the structure and order of the GaN-GaNa solid liquid interface during GaN growth.

\section{References}

[1] H. Reichert, M. Denk, J. Okasinski, V. Honkimäki, H. Dosch, Giant metal compression at liquid-solid (pb-si, in-si) schottky junctions, Phys. Rev. Lett. 98 (2007) 116101. doi:10.1103/PhysRevLett.98.116101.

[2] W. D. Kaplan, Y. Kauffmann, Structural order in liquids induced by interfaces with crystals, Annual Review of Materials Research 36 (1) (2006) 1-48. doi:10.1146/annurev.matsci.36.020105.104035.

[3] W. Huisman, J. Peters, M. Zwanenburg, S. de Vries, T. Derry, D. Abernathy, J. van der Veen, Layering of a liquid metal in contact with a hard wall, Nature 390 (6658) (1997) 379-381. doi: $10.1038 / 37069$.

[4] M. F. Reedijk, J. Arsic, F. F. A. Hollander, S. A. de Vries, E. Vlieg, Liquid order at the interface of kdp crystals with water: Evidence for icelike layers, Physical Review Letters 90 (2003) 066103. doi:10.1103/PhysRevLett.90.066103.

[5] H. Reichert, V. Honkimäki, A. Snigirev, S. Engemann, H. Dosch, A new X-ray transmission-reflection scheme for the study of deeply buried interfaces using high-energy microbeams, Physica B: Condensed Matter 336 (1) (2003) 46-55. doi:10.1016/S0921-4526(03)00268-0.

[6] V. Honkimäki, H. Reichert, J. S. Okasinski, H. Dosch, Xray optics for liquid surface/interface spectrometers, Journal of Synchrotron Radiation 13 (6) (2006) 426-431. doi:10.1107/S0909049506031438.

[7] H. Reichert, O. Klein, H. Dosch, M. Denk, V. Honkimäki, T. Lippmann, G. Reiter, Observation of five-fold local symmetry in liquid lead, Nature 408 (2000) 839-841. doi:10.1038/35048537.

[8] D. Calzolari, D. Pontoni, M. Deutsch, H. Reichert, J. Daillant, Nanoscale structure of surfactant-induced nanoparticle monolayers at the oil-water interface, Soft Matter 8 (45) (2012) 11478-11483. doi:10.1039/c2sm26520f.

[9] F. J. Wirkert, M. Paulus, J. Nase, J. Möller, S. Kujawski, C. Sternemann, M. Tolan, X-ray reflectivity measurements of liquid/solid interfaces under high hydrostatic pressure conditions, Journal of Synchrotron Radiation 21 (1) (2014) 1-6. doi:10.1107/S1600577513021516.

[10] F. Venturini, S. Schoder, W. Kuhs, V. Honkimaki, L. Melesi, H. Reichert, H. Schober, F. Thomas, A large-volume gas cell for high-energy X-ray reflectivity investigations of interfaces under pressure, Journal of synchrotron radiation 18 (2) (2011) 251256. doi:10.1107/S0909049510052106.

[11] P. Bernard, K. Peters, J. Alvarez, S. Ferrer, Ultrahigh vacuum/high pressure chamber for surface X-ray diffraction experiments, Review of Scientific Instruments 70 (2) (1999) 14781480. doi:10.1063/1.1149609.

[12] E. Vlieg, Surface and Interface Science, Vol. 1: Concept and Methods, Wiley-VCH, 2012, Ch. 3.4.2, pp. 375-425.

[13] R. Felici, Characterization of Materials, John Wiley \& Sons, Inc., 2012. doi:10.1002/0471266965.com079.pub2.

[14] M. Ruat, C. Ponchut, Characterization of a Pixelated CdTe $\mathrm{X}$-Ray Detector Using the Timepix Photon-Counting Readout Chip, IEEE Transactions on Nuclear Science 59 (5) (2012) 2392-2401. doi:10.1109/TNS.2012.2210909.

[15] E. Vlieg, Integrated intensities using a six-circle surface x-ray diffractometer, Journal of Applied Crystallography 30 (1997) 532-543. doi:10.1107/S0021889897002537.

[16] F. Kawamura, T. Iwahashi, K. Omae, M. Morishita, M. Yoshimura, Y. Mori, T. Sasaki, growth of a large GaN single crystal using the liquid phase epitaxy (LPE) technique, Japanese journal of applied physics 42 (1AB) (2003) L4-L6. doi:10.1143/JJAP.42.L4. 
[17] F. Kawamura, M. Morishita, K. Omae, M. Yoshimura, Y. Mori, T. Sasaki, The effects of na and some additives on nitrogen dissolution in the ga-na system: A growth mechanism of gan in the na flux method, Journal of Materials Science: Materials in Electronics 16 (1) (2005) 29-34. doi:10.1007/s10854-005-4955-8.

[18] DJP project number 31190-oc-02a, http: //www.djp.fr/.

[19] S. Park, C. Cho, S. Kim, S. Jeong, Growth and impurity study of gan single crystal grown by na flux, Journal of the Korean Physical Society 42 (2003) S161-S164.

[20] M. Razeghi, The MOCVD Challenge: A survey of GaInAsPInP and GaInAsP-GaAs for photonic and electronic device applications, CRC Press, 2010.

[21] T. Itami, M. Shimoji, J. Meijer, G. Vinke, C. van der Marel, W. van der Lugt, The phase diagram and the electrical resistivity of liquid na-ga alloys, Physica B+C 142 (2) (1986) 145 - 151. doi:10.1016/0378-4363(86)90206-8.

[22] H. Yamane, D. Kinno, M. Shimada, F. J. Disalvo, Crystal growth of gan from na-ga melt in bn containers, Journal of the Ceramic Society of Japan 107 (1250) (1999) 925-929. doi:10.2109/jcersj.107.925. 